\section{Cahiers de littérature orale}

$66 \mid 2009$

Mémoire des CLO

\title{
La parole pilée : accès au symbolisme chez les Gbaya 'Bodoé de Centrafrique
}

Paulette Roulon-Doko et Raymond Doko

\section{OpenEdition}

1 Journals

Édition électronique

URL : https://journals.openedition.org/clo/656

DOI : $10.4000 /$ clo. 656

ISSN : 2266-1816

Éditeur

INALCO

Édition imprimée

Date de publication : 1 janvier 2009

Pagination : 217-232

ISBN : 978-2-85831-188-0

ISSN : 0396-891X

Référence électronique

Paulette Roulon-Doko et Raymond Doko, "LA PAROLE PILÉE : ACCÈs AU SYMBolisme CHEZ LeS GBAYA 'Bodó́ De CENTRAFRIQue ", Cahiers de littérature orale [En ligne], 66 | 2009, mis en ligne le 25 février 2013, consulté le 01 juillet 2021. URL : http://journals.openedition.org/clo/656 ; DOI : https://doi.org/ $10.4000 /$ clo.656

Ce document a été généré automatiquement le 1 juillet 2021.

\section{(c) (1) (9)}

Cahiers de littérature orale est mis à disposition selon les termes de la Licence Creative Commons Attribution - Pas d'Utilisation Commerciale 4.0 International. 


\title{
La parole pilée : accès au symbolisme chez les Gbaya 'Bodoé de Centrafrique
}

\author{
Paulette Roulon-Doko et Raymond Doko
}

\section{NOTE DE L'ÉDITEUR}

Article paru pour la première fois dans Cahiers de littérature orale, $\mathrm{n}^{\circ} 3,1977$, p. 33-49.

1 Appartenant à une culture de traduction orale, les Gbáyá bòdò accordent une grande importance à la parole wèn ${ }^{1}$. Son acquisition par le jeune enfant marque le point de départ du cycle de la connaissance (Roulon, 1980 : 101-104) et son maniement constitue un savoir très valorisé. Selon les moments la parole devient une conversation jéré, dispute Pàrì ; querelle kpérì, jugement kità, blague zèí, insulte dàrà, etc. Mais quelles qu'en soient les conditions d'émission, elle réfère toujours à deux niveaux distincts : la parole ordinaire géé wèn (simple/parole) dont le sens colle à ce qui est dit, et la parole profonde dúká wèn (profonde/parole) dont il faut chercher le sens au-delà des mots. Pour cette dernière, tous les éléments du discours ne sont pas porteurs de l'intention réelle qui sous-tend l'ensemble. Le récepteur doit savoir ne pas s'attarder aux mots vides pàyá wèn (déchets/paroles) pour mettre à nuํㄹ ${ }^{2}$ les éléments qu'une réflexion attentive lui permettra alors de comprendre. Le locuteur « prépare » cette parole, veille à ce qu'elle ait du « goût » et pour ce faire y met des « paroles pilées » tó-wèn ${ }^{3}$.

2 Piler du sésame, par exemple, c'est l'écraser en une masse dont les éléments de base les graines - ne sont plus discernables. De même un tó-wèn est un passage du discours où les éléments constitutifs - les mots - ne sont plus compréhensibles tels quels. L'interlocuteur va devoir chercher, au-delà des mots, la signification réelle de ce qu'il a entendu. Obscurcissement volontaire du sens, dissimulation soigneusement préparée, ces tó-wèn sont l'expression même de l'habileté du locuteur à manier la parole, la 
manifestation de son aisance à en maitriser le niveau profond, le signe de son savoir dire.

3 Tout procédé qui établit une distance entre ce qui est dit et ce qu'il y a à comprendre participe du même mécanisme de pensée qui consiste à "cacher la parole " húsá wèn. Aussi est-ce en suivant les étapes que franchit l'enfant puis l'adolescent pour atteindre au maniement de la parole profonde que l'on pourra saisir comment s'acquiert ce processus de raisonnement qui exprime la logique fondamentale de tout usage élaboré de la parole chez les 'Bodoé.

\section{Apprentissage}

Dès quatre ans, lorsque l'enfant parle bien et comprend ce qu'on lui dit en y répondant correctement, son père ou sa mère va, en situation, le conseiller. Leur mise en garde invite toujours l'enfant à prendre conscience du déroulement de l'action et de son aboutissement logique. Par exemple ${ }^{4}$, si un enfant coupe un fruit avec un couteau trop grand pour lui et avec lequel de surcroît il s'y prend mal, on ne le lui retire pas, comme on l'aurait fait avec un plus petit, mais on l'avertit :

- zók tòk nè fók tè ?Érméií!

vois/sang/qui/coule/à/main.ta.cette

- vois le sang qui coule de ta main là !

S'il rétorque :

- tòk bé ná

sang/peut être/pas

$-\mathrm{y}$ a pas de sang!

le dialogue se poursuit ainsi :

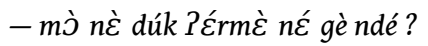

chose/qui/reste/main-ta/est/quoi/est-ce que

- qu'est-ce que tu as en main?

$-n \varepsilon ́$ pàyà

est/couteau

- c'est un couteau

$-h \grave{\varepsilon} \varepsilon \dot{\varepsilon} \dot{\varepsilon} !$

- ah bon!

On en reste là. Si l'enfant persévère et se blesse, l'adulte conclut en montrant sa blessure : «y a pas de sang ». Ce procédé pédagogique responsabilise très tôt l'enfant et l'incite à faire attention aux paroles qu'on lui adresse.

6 Peu après, les parents vont intentionnellement «emmêler la parole " yúkútá wèn, y faire un nœud que l'enfant devra défaire pour saisir ce qui lui échappe au premier abord. Il accède, ce faisant, au domaine des paroles cachées. Cela commence toujours par des situations peu ambiguës. Par exemple, s'il trébuche sur quelque chose, l'adulte contredisant le réflexe qui le poussera à regarder par terre, lui dira au choix :

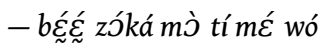
refuser/regarder/chose/devant/toi/vraiment

- surtout de refuser de regarder devant toi!

- yák-té-zòk mò tí mé ná wó!

il ne faut regarder/chose/devant/toi/pas/vraiment

- surtout il ne faut pas regarder devant toi!

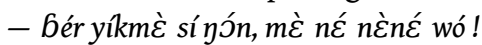

lève/yeux-tes/vers/haut/tu/vas/avec/vraiment

- lève les yeux au ciel, et surtout vas-y! 
7 Toutes ces phrases ont une courbe mélodique identique en finale avec un ton suprahaut rendu dans la traduction par «surtout ». C'est une marque formelle qui attire l'attention de l'enfant sur la nature du message qui lui est adressé.

8 Par la suite, toutes les petites commissions que l'on fait volontiers faire à l'enfant seront accompagnées de ce type de parole. Lorsque son père l'envoie chercher un peu de tabac chez un voisin, il recommandera :

- né nèmè dé sàà hớgó, sćkómé pèá đòy!

va/que-tu/fais/jeu/comme ça/avant que-tu/reviens/derrière

- surtout va bien t'amuser avant que de revenir!

et sa mère, en mettant de côté ce qu'elle vient de cuire alors qu'elle part pour les champs, lui conseillera :

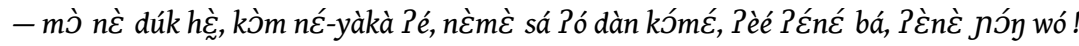
chose/qui/reste/ici/si-je/hypothétique-pars/déjà/que-tu/appelles/les/amis/detoi/puis/vous/prenez/vous/mangez/vraiment

- ce qui reste là, quand je serai partie, appelle tes amis, prenez-le et surtout mangez-le!

Enfin, l'enfant pris en flagrant délit de bêtise se fait ainsi admonester :

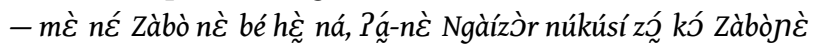

cela/est/ /qui/peut être/ici/pas/c'est que/ /broie/paille/de/ -ce

- voici surtout Zabo qui n'est pas là, que Ngaizor écrabouille la paille de Zabo !

9 Le père se nomme Ngaizor et le fils, qui joue sans scrupules dans la paille, se nomme Zabo. Or le père n'a assurément pas à attendre l'absence de son fils pour faire quelque chose, par contre quand le père n'est pas là, l'enfant en profite.

10 Toutes ces recommandations procèdent par inversion ziká wèn (retourner/parole) ${ }^{5}$ : il y a distorsion entre la réalité et la parole émise, la parole prend systématiquement le contre-pied de ce qui est effectivement souhaité. Elles créent, chez l'enfant, une réflexion sur le réajustement nécessaire à opérer entre le dire et le vouloir-dire du locuteur.

\section{Introduction des tó-wèn}

11 Progressivement, l'adulte laisse tomber la courbe d'intonation qui marque ces énoncés. Puis ne conservant que le procédé d'inversion auquel l'enfant est déjà bien rodé, il introduit un premier tó-wèn. Celui-ci, souvent entendu dans la bouche des grands-mères pour sermonner leurs petits-enfants, est presque un calque de la remontrance précédemment citée :

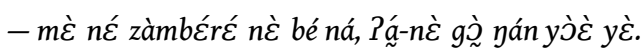

cela/est/guib/qui/peut être/ici/pas/c'est que/panthère/ parade/danse/en effet

- comme le guib n'est pas là, la panthère en effet fait une parade de danse ${ }^{6}$.

12 Ce tout premier tó-wèn est unique et occupe, de ce fait, une position charnière entre ces deux niveaux de la parole cachée que sont les simples conseils et les véritables tó-wèn. Des premiers il conserve le procédé de l'inversion et comme les seconds il se caractérise entre autres par l'emploi d'éléments sans rapport direct avec la situation réelle. Ce sont ici le guib zàmbérć, animal inoffensif, et la panthère gò , animal féroce. Leur association crée une corrélation sémantique qui renvoie à la notion de force.

Pendant tout ce processus d'acquisition des paroles cachées, il n'y a jamais d'explication donnée. Dans tous les cas, c'est sa propre réflexion que l'enfant doit solliciter pour faire la part des choses et saisir ce qu'on attend de lui. Si l'enfant, par 
exemple, ne comprend pas le sens de ce premier tó-wèn et demande que l'adulte le lui répète, celui-ci coupe court en disant :

- mî̀ ndé, bè̀ Pà dé bèe héć ndál gbérè wó!

moi/que/saison sèche/elle/fait/vite/que-nous/sucions/Aframomum/vraiment

- je dis que la saison sèche arrive vite que nous nous régalions de fruits

d'Aframomum

14 À moins qu'il ne se contente d'en revenir à la simple inversion, en précisant le nom des acteurs visés.

Ainsi préparé depuis l'enfance à être attentif aux paroles qu'on lui dit, l'adolescent est bientôt en mesure de repérer certains tó-wèn parmi ceux qui assaisonnent le discours des adultes. Il reçoit tout particulièrement des conseils sous la forme de tó-wèn qui reviennent facilement à son propos :

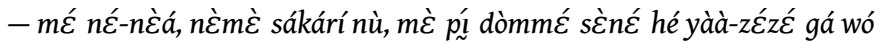

tu/hypothétique-vas/que-tu/creuses/terre/tu/jettes/queue-ta/dedans/comme/

sauterelle sp./comme/vraiment

- si tu y vas, fais un creux dans le sol, mets-y ta queue comme la sauterelle yàà-

zغ́zé.

16 Yàà-zÉzÉ est une petite sauterelle que tous les enfants connaissent bien puisqu'ils l'attrapent volontiers, surtout lorsqu'elle est absorbée dans sa ponte. Les deux éléments, A : faire un trou et B : s'y poser, constituent une corrélation, qui explicite la valeur de yàà-zćzź en tant que symbole de toute activité absorbante. L'enfant comprend que, dans son cas personnel, c'est le jeu qui est visé et qu'il lui est conseillé, toujours par inversion, de se dépêcher, de ne pas se laisser distraire en route.

Deux avertissements s'adressent expressément aux jeunes gens qui se font remarquer par leur mauvaise conduite. Le premier :

- mé té-kpà déy ká wár ná

tu/virtuel-trouves/plante magique/à côté/route/pas

- tu ne trouveras pas de plante magique sur le bord du chemin

s'emploie aussi bien pour un garçon que pour une fille. La plante magique dén, symbolise ici le remède universel, représentant pour un adolescent le conjoint grâce auquel il quitte le célibat, lequel est ressenti, s'il dure trop longtemps, comme une infirmité sociale. La mention « sur le bord du chemin » marque la proximité spatiale. Ce tó-wèn signifie pour l'adolescent ou l'adolescente en question que son comportement lui attire la désapprobation de tous et qu'en conséquence il ne trouvera personne ici qui accepte de l'épouser. Dans des circonstances analogues, une jeune fille pourra s'entendre dire :

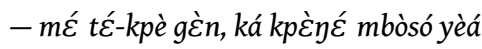

tu/virtuel-fermes/nasse/alors/sans manche/machette/est/entrée

- tu poseras une nasse et une machette démanchée y entrera

La nasse représente toute activité qu'on prépare longuement, et la machette sans son manche évoque un pénis. La jeune fille est ainsi mise en garde : à force de se tisser une mauvaise réputation, elle se retrouve réduite à une situation périlleuse. Les relations sexuelles expriment ici une activité qui transforme la jeune fille et peut, dans certains

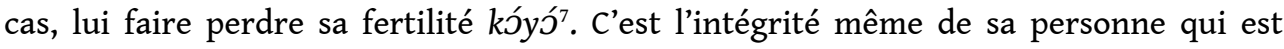
menacée et non sa virginité, laquelle n'est pas prise en considération.

Enfin, les enfants commenceront à utiliser entre eux certains tó-wèn. Par exemple, lorsqu'un enfant est insulté par un de ses camarades qui a profité de ce qu'il arrivait chez lui pour se protéger dans son foyer, il attendra - dans l'incapacité immédiate de 
lui répondre - l'occasion de se retrouver à égalité avec lui. Ce jour-là, il l'abordera ainsi :

- gbàdidì gòná dỡo yàà kéí

guêpe maçonne/a coupé/dos/grand-mère/de-lui

- la guêpe maçonne menace sa grand-mère

21 La guêpe, par son bourdonnement, symbolise la parole incompréhensible, le murmure indéchiffrable. La grand-mère renvoie à l'insulte antérieure, la parole qui fonde l'explication présente. De cette manière l'enfant signifie que le rapport de force qui a joué en faveur de l'insulteur, le contraignant à se contenter de grommeler entre ses dents, n'existe plus aujourd'hui et qu'il va enfin pouvoir répondre ouvertement, se vengeant ainsi de l'insulte passée.

\section{Conditions d'emploi}

L'ensemble des tó-wèn fixés par l'usage, ceux qui reviennent toujours plus ou moins sous la même forme dans les conversations, soit plus de trois cents énoncés pour notre corpus, ne constitue pas un savoir récitable en tant que tel. Personne ne peut, sur simple commande, dire des tó-wèn tandis que chacun connaît des contes tò, des devinettes sìn, pour lesquels il peut hors contexte dénombrer l'importance de son répertoire personnel ${ }^{8}$. On ne peut recueillir ces tó-wèn qu'en situation, en les saisissant dans un discours ${ }^{9}$. Contrairement aux contes et aux devinettes qui pourraient eux se définir comme des paroles de nuit, les tó-wèn ne créent pas un genre, mais sont une façon de dire. Si en conversant avec quelqu'un, on ne veut pas être compris par un tiers qui surviendrait, il suffira de "piler» le reste de ce qu'on avait à dire afin que le nouveau venu ne saisisse pas le sens de la conversation. Dire à son interlocuteur «pilons seulement!» Pèc̀ tó tòó Pèá! l'avertit immédiatement de l'arrivée d'un importun. Ce dernier peut très bien réaliser ce qui s'est passé, mais la politesse l'empêchera de poser la moindre question..., à lui de démêler le sens de ce qu'il a entendu dans la mesure où il possède suffisamment d'éléments.

Le maniement et le rôle de la parole dans les relations sociales sont ici fondamentaux pour expliquer la généralisation du processus de dissimulation de la parole. Ne pas dire les choses explicitement, ne pas appeler un chat un chat, sont les bases mêmes d'un bon comportement en société. En effet, un 'Bodoé ne doit jamais, par ses paroles, attirer la honte foyò sur quelqu'un, et pour ménager la sensibilité d'autrui il ne faut donc pas

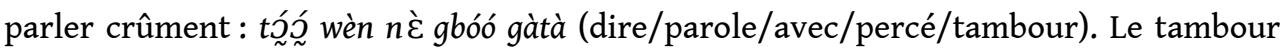
sur pied à une seule membrane gàtà est dans les contes un véritable cheval de Troie, dissimulant des chiens pour les introduire chez les aulacodes ${ }^{10}$, escamotant l'enfant désobéissant. L'image du tambour percé est éloquente : on voit tout ce qui aurait pu s'y trouver dissimulé. Cette parole directe, sans sous-entendus, est réservée aux causeries qu'échangent mari et femme dans l'intimité. En société, la manière dont on parle est plus importante que ce que l'on dit. Cela est également vrai pour les messages rapportés. L'enfant envoyé en commission n'en doit pas délivrer tel quel le message qui lui a été confié. Il convient de ne pas déranger intempestivement le destinataire. Le messager se met dans un coin, se fait une idée de ce qui occupe celui qu'il vient voir, attend dans tous les cas qu'il ait terminé ce qu'il est en train de faire ${ }^{11}$. S'il est directement questionné, il répond mò bé ná « il n'y a rien ». Puis quand il pense qu'il ne dérange plus, il se dirige vers celui à qui on l'a adressé et lui parle à voix basse. 
Chuchoter, faire des apartés fúná wèn (chuchotée/parole) est l'expression même de ce qu'il faut faire. Dans une conversation, savoir "piler la parole » c'est respecter de la même façon les convenances. Ce qui est dit ainsi ne choque personne: chacun interprète le tó-wèn en fonction des éléments qui sont en sa possession, celui qui est visé l'est de façon anonyme.

\section{Structuration et mécanisme de fonctionnement}

Chaque tó-wèn comprend deux éléments, chacun perçu comme incomplet. Le terme gbáá gúrú wèn (fendu/moitié/parole) " parole fendue par moitié » qui les désigne rend bien compte de la conception même du locuteur. Ce même terme gbáá gúrú peut s'appliquer à chaque moitié de la coque des arachides ou des graines de courges. Ces deux éléments de paroles se réfèrent l'un à l'autre et créent une corrélation qui, elle, définit un symbole. Par exemple :

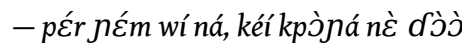

lien/convient/homme/pas/alors-on/ôte/par/dessous

- si une ceinture ne va pas, on la retire en la laissant glisser

5 Les deux moitiés de parole séparées: A "ne va pas » et B «on l'ôte par le bas ", s'assemblent pour définir la ceinture comme étant le symbole de ce qui doit être à la mesure convenable, adapté aux besoins. Cette structure binaire sur laquelle s'organise le tó-wèn propose une corrélation sémantique qui renvoie toujours à un symbole qu'elle définit en quelque sorte. La masse des tó-wèn fixés par l'usage est, par conséquent, le corpus qui permet l'identification des symboles et donne pour chacun d'eux les éléments qui le définissent. Cela compose une sorte de dictionnaire du symbolisme 'bodoé que chacun intègre peu à peu à son savoir. Il n'est évidemment pas question pour quiconque de le réciter, mais d'en disposer à sa guise, comme nous allons le voir par la suite.

Repérer un tó-wèn et l'interpréter réclame toujours une attention, puis une réflexion. Un tó-wèn souvent entendu et dont la référence symbolique est aussitôt saisie n'est pas pour autant totalement compris. Le symbolisme exprimé par le tó-wèn doit toujours être rapporté à l'individu et aux relations dans lesquelles il est impliqué. Les 'Bodoé disent d'ailleurs du tó-wèn qu'il est comme "la maison de la guêpe maçonne » tùá gbàdìdì. En effet, lorsque l'on casse l'alvéole de terre façonnée par cette guêpe, au lieu des seuls œufs de guêpe auxquels on s'attendait, on trouve des larves, des mouches, voire une araignée. De la même façon, un tó-wèn va donner lieu à une pluralité d'interprétations selon la situation de chacun ; mais toutes ces interprétations possibles doivent manifester la corrélation symboliquement exprimée par ce tó-wèn.

Un tó-wèn reste dans la tête de celui qui l'a entendu jusqu'à ce qu'il en ait déchiffré le sens. Parfois un adolescent cherchera à consulter l'un de ses parents lorsqu'il n'arrive pas à déchiffrer le sens d'un tó-wèn qui lui a directement été adressé. Ainsi un adolescent dont le vélo avait été abîmé par un de ses camarades à qui il l'avait prêté, s'entendit répondre par ce dernier à qui il réclamait le prix de la pièce cassée :

- Péí dé-mó-fàn dèrè, kéi bàá gbà?áăa pí nè dòj̀ sćkéi sèmà mbéà gònáà. on/imminent-tresse/natte/alors-on/a pris/ancienne/mise/en/dessous/avant queon/pose les brins/nouvelles/dessus-son

- quand on s'apprête à tresser une natte, on pose la natte usagée dessous, avant que de poser les brins de la nouvelle dessus. 
Ayant cherché vainement ce que l'autre voulait dire par là, il demanda conseil à sa mère. Celle-ci lui suggéra alors de se rappeler s'il n'avait pas, lui-même, il n'y avait pas si longtemps, abîmé le vélo de son camarade? Il comprit que l'autre rejetait sa demande et l'invitait à réfléchir sur les deux vélos dont les états étaient mis en parallèle. Pourquoi ne retenait-il que l'état de son seul vélo! La natte symbolise la permanence en dépit du temps, l'équivalence de deux états que seule la marque du temps distingue : la natte usagée et la nouvelle.

Le mécanisme qui permet de «cacher la parole » débute par le déchiffrage du langage inversé où la parole émise contredit le déroulement logique auquel on a rendu très tôt l'enfant attentif (cf. § Apprentissage). Il s'agit de repérer toute distorsion entre ce qui est dit et ce qui est voulu. L'introduction d'éléments sans rapport avec le reste du discours marque l'apparition des premiers tó-wèn. L'attention est portée sur toute portion d'énoncé sans signification apparente. On pourrait, pour mémoire, signaler qu'il existe des énoncés, en nombre réduit, qui comportent un terme qui, lui aussi, n'a pas de signification apparente, soit parce qu'il est créé de toutes pièces, soit parce que son sens ne peut être rapporté à son usage normal. Cela crée des expressions ${ }^{12}$ qui ne se prêtent pas à une analyse, mais nécessitent qu'on les mémorise tels quels. Ainsi :

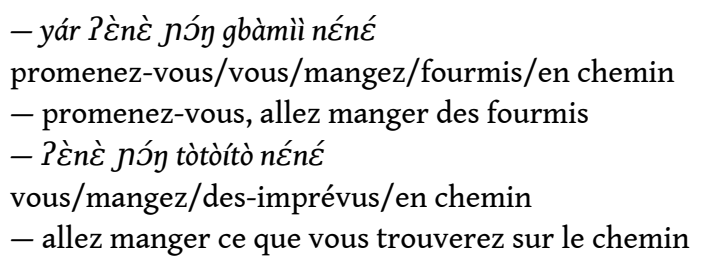

Ces deux énoncés s'adressent aux enfants qu'on veut aller voir jouer, faire ce qui leur plait, plus loin.

- mé jòyá fió dǒk-tòrò

tu/as mangé/mortes/chenilles/roseaux

- tu as mangé des larves de roseaux mortes

Cette expression signifie que celui qui parle et à qui l'on s'adresse ainsi n'arrive pas à rester sur un même sujet, qu'il saute d'un sujet à l'autre comme un papillon !

Ce rappel n'est pas inutile, car il montre bien que si le repérage de telles expressions s'effectue sur la même base d'identification des éléments obscurcissant le sens du message, la nature même de ces éléments est fondamentalement différente et détermine le traitement que chacun d'eux reçoit. D'une part, il n'y a qu'un seul foyer (terme ou groupe de termes) qui fait obstacle à la compréhension: il n'y a qu'à se le faire expliquer et à le retenir. C'est le cas des expressions présentées ci-dessus. D'autre part, il y a dans l'énoncé deux foyers, ce sont les "paroles fendues par moitié » qu'il faut relier entre elles, car elles sont solidaires : là, il n'y a pas d'explication à demander ; il convient de procéder à une analyse, à un décodage. La présence de cette structure binaire apparaît comme la caractéristique fondamentale de tout tó-wèn.

Un certain nombre d'énoncés, fixés par l'usage et qu'on entend souvent, semblent à première vue être des tó-wèn, tel :

- wéwéi né gbà-yik

homme/est/œil

- l'homme est un œil

autrement dit, tout homme doit voir clair, faire la part des choses. En fait, cet énoncé n'est pas retenu comme tó-wèn par les locuteurs. En fin de compte ils le nomment 
"parole dite» tỡ apparemment de celui-ci, l'énoncé suivant, en revanche, est bien un tó-wèn :

- wéwéi né zày táná, Pèì nóy, Péínj̀jà bé tà

homme/est/tripes/tortue/on/mange/on/a mangé/petites/pierres

- l'homme est une tripe de tortue, on la mange, on mange des petits cailloux

L'élément «tripes de tortue " qui représente une nourriture fort appréciée des hommes, est mis en relation avec l'élément " petits cailloux "- il peut se trouver qu'un petit caillou soit resté - qui représente un élément désagréable. Ces deux moitiés de parole volontairement séparées l'une de l'autre, se rassemblent pour symboliser l'homme comme étant ce qui ne peut se juger de l'extérieur, celui dont la force est à l'intérieur, cachée des regards.

\section{Création individuelle}

Ayant saisi la façon dont s'organise tout tó-wèn, le locuteur va pouvoir créer selon les besoins de sa conversation ses propres tó-wèn. Pour cela il se conforme à une structure binaire et puise par ailleurs des symboles dans ce réservoir que constituent les tó-wèn fixés par l'usage ou du moins dans la partie de ceux-là que sa mémoire a engrangée. Il ne s'agit pas, dans ce cas-là non plus, de mémoriser le tó-wèn qu'on aura dit dans telle occasion. C'est l'adéquation des symboles utilisés à la situation et l'habileté du locuteur à insérer ce tó-wèn dans le contexte linguistique, qui sont ici manifestés. Quelqu'un qui ne parviendrait pas à placer à l'occasion un tó-wèn du fonds commun, à défaut d'en créer lui-même, est très mal considéré. Son discours plat, fade n'intéresse personne tandis que la parole profonde maniée avec aisance est appréciée de tous, voire recherchée car elle donne matière à réflexion, elle a du poids. Mieux vaut un tó-wèn employé à bon escient qu'un long discours où il n'y a à comprendre que ce que disent les mots.

Nous ne donnerons qu'un exemple d'une création individuelle de tó-wèn et illustrerons à ce propos tout ce qu'un tel usage de la parole apporte sur le plan social.

Un jeune homme s'entend dire ceci :

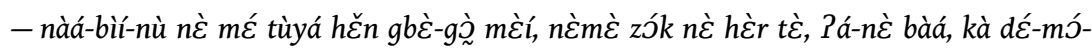

mbذ̀r.

piège à souris/que-tu/as posé/à/nom d'une rivière/là-en question/que-tu/vois/ avec/attaché/corps/c'est/que/a pris/alors/imminent-pourrit

- le piège à souris que tu as posé à Gbè-gon là-bas, que tu relèves avec paresse, ça a

pris et ça va pourrir.

Le piège à souris nàá-bii-nù, comme l'ensemble des pièges, qu'ils soient à lacet pér, à assommoir sàk, ou à poisson gغ̀n "nasse ", symbolise toute activité qui demande une longue préparation, une activité suivie. En l'occurrence c'est le mariage du jeune homme qui est visé : la période de fiançailles étant longue et chargée d'obligations du gendre vis-à-vis de ses beaux-parents. Il comprend donc qu'il s'agit d'une critique de son attitude jugée paresseuse. Les deux éléments en corrélation, d'une part «ça a pris ", et de l'autre "ça va pourrir ", servent ici à spécifier le point de vue de celui qui parle. En un mot, la jeune fille qu'il courtise (gibier de piège) risque fort ainsi que sa famille de se lasser (va pourrir) d'un prétendant si peu régulier dans ses visites et qui s'illustre fort peu dans les tâches qui lui incombent. Une telle critique dite par le biais d'un tó-wèn interdit toute protestation que l'intéressé n'aurait pas manqué de faire si 
on avait tenté de lui dire directement « tu es vraiment trop paresseux... ». Cela ne crée aucune gêne, les autres auditeurs n'étant pas au courant comme lui de ses occupations et la politesse imposant dans tous les cas à chacun de ne faire aucune réflexion, de ne poser aucune question qui puisse traduire le message en clair.

D'une manière générale quelqu'un à qui on adresse un tó-wèn peut répondre par le même procédé, s'il comprend sur-le-champ de quoi il s'agit. Dans l'exemple présenté, il n'y a aucune réponse à faire. Quand une réponse s'impose - quelqu'un qui sert d'intermédiaire par exemple -, elle peut fort bien être différée dans le temps. Cependant si elle tarde trop, l'intéressé reviendra à la charge en demandant à l'autre "le message que j'ai transmis l'autre jour, est-ce que ça revient?... ». Au besoin, il redira son message. Il est certain que de tels échanges ont lieu dans une société où il n'existe aucune contrainte de temps. Chacun y prend le temps de vivre à sa guise, à son rythme, et que la communication soit lente, dans bien des cas, importe peu.

\section{Conclusion}

41 La définition que donne le Petit Larousse illustré de 1906 du proverbe - maxime exprimée en peu de mots et devenue populaire - ne saurait s'appliquer telle quelle au tó-wèn. En effet, ce dernier n'est pas caractérisé par une formulation brève, mais par une structure binaire ${ }^{14}$ et il ne concerne pas les seuls énoncés que l'usage a fixés, de façon plus ou moins variable dans le détail, puisque tout l'art de parler consiste en outre à pouvoir créer selon ses besoins ses propres tó-wèn.

Le tó-wèn, manifestation par excellence de la parole profonde, constitue une voie royale pour l'accès au symbolisme de cette culture. Le réservoir de symboles que représentent tous les tó-wèn fixés par l'usage permet la création de tó-wèn individualisés et donne des clés pour interpréter les rêves. Les 'Bodoé sont en effet très attentifs aux rêves qu'ils peuvent faire et s'attachent avec beaucoup de soin à l'interprétation de ce langage dont ils tiennent à déchiffrer les éléments afin d'en comprendre le sens sous-jacent. Cette distance qui sépare le rêve du message qu'il délivre est de même nature que celle qui, dans le tó-wèn, sépare ce que l'on dit de ce que l'on veut dire. N'affirment-ils pas d'ailleurs que le tó-wèn est le rêve de la parole?

\section{BIBLIOGRAPHIE}

CALAME-Griaule, Geneviève, 1965, Ethnologie et langage, la parole chez les Dogon, Paris, Gallimard, Bibliothèque des sciences sociales. Cf. chap. V « L'art de la parole », p. 446 et sq.

CAUvin, Jean, 1976, Préalables à une recherche parémiologique, Afrique et langage, nº 5, p. 5-34.

EVANS-PRITCHARD, Edward, E., 1963, Meaning in Zande proverbs, Man, vol. LXIII, pp. 4-7.

EVANS-PRITCHARD, Edward, E., 1964, Zande proverbs: final selection and comments, Man, vol. LXIX, pp. 1-5. 
GoufFÉ, Claude, 1981, Comment recueillir et éditer les proverbes haoussa, quelques suggestions pratiques, Itinérances... en pays peul et ailleurs II, Mémoires de la société des Africanistes, p. 115-135. GREIMAS, Algridas Julien, 1970, Du sens, essais sémiotiques, Paris, Seuil, p. 309-314 (en particulier le chapitre « Les proverbes et les dictons »).

JAKOBSON, Roman, 1966, Essais de linguistique générale, Paris, Éditions de Minuit, p. 30 et sq.

PENEL, Jean-Dominique, 1975, Quelques aspects du langage indirect chez les Zandé du Haut-Mbomou, Bangui, Centre protestant pour la jeunesse, $114 \mathrm{p}$.

PENEL, Jean-Dominique, 1977, Proverbes centrafricains et philosophie, texte dactylographié, 13 p.

Roulon, Paulette, 1980, La conception gbaya du corps humain, Journal des Africanistes, 50/1, p. 59-106.

Roulon, Paulette, 1981, Les rites de fécondité chez les Gbaya kara, Itinérances... en pays peul et ailleurs II, Mémoires de la société des Africanistes, p. 355-377.

SZEMERKÉNYI, Agnès, 1981, L'utilisation des proverbes, Cahiers de littérature orale, nº 9, p. 79-104.

\section{NOTES}

1. Tout au long de cet article les termes gbaya 'bodoé, éventuellement précisés par un mot à mot mis entre parenthèses, sont placés immédiatement après les termes français auxquels ils correspondent. Dans le cas d'expressions dépassant le mot, celles-ci sont placées entre guillemets pour éviter toute difficulté de repérage. Par ailleurs, les énoncés gbaya 'bodoé sont toujours accompagnés d'un mot à mot où chaque terme est isolé par des barres obliques, le tiret signalant un groupement ou un amalgame. Aucune considération grammaticale n'est introduite dans ce mot à mot qui n'a pour but que de permettre un accès simple à la structuration gbaya.

2. 6Éfá wèn "décortiquer la parole ", ce même verbe s'applique pour le décorticage des graines de courges ou des élytres de sauterelles ou de coléoptères comestibles.

3. Dans la suite de cet article, nous avons préféré conserver ce terme tó-wèn plutôt que de le traduire approximativement par "proverbe", terme qui a une charge sémantique trop spécifique dans l'usage qui en est fait en français et pour lequel il n'est pas question ici d'en faire l'analyse en tant que métalangue.

4. Il s'agit toujours de situations où le danger reste limité à un bobo sans gravité.

5. Les locuteurs précisent bá zú wèn Pé há domáà, bá dòmáa Pé há zúa (prends/tête/parole/pose/ à/queue-sa/prends/queue-sa/pose/à/tête-sa) «mets la tête de la parole à la queue, mets sa queue en tête ".

6. Cet énoncé est comparable à l'inversion de « quand le chat n'est pas là les souris dansent ». Ce tó-wèn est bien réservé aux jeunes, les adultes entre eux utilisent pour dire la même chose un autre tó-wèn.

7. Pour plus d'explications, on se reportera à Roulon (1981).

8. Remarquons que le conte tò et la « devinette " sìn sont des termes simples, inexplicables tandis que tó-wèn " parole pilée » est un composé qui marque le résultat d'une action, le pilage.

9. La plupart des auteurs qui ont travaillé sur l'Afrique noire ont remarqué une situation analogue, cf. ouvrages cités dans la bibliographie.

10. L'aulacode, Thryonomys swinderianus (Temminck), est un rongeur très apprécié comme gibier.

11. Ceci concerne surtout le repas : il ne convient pas d'interrompre quelqu'un qui mange.

12. Du même type que les expressions dites populaires, telle « du coq à l'âne » que l'utilisateur moyen ne peut expliquer. 
13. Ce terme est repris dans le sens que lui donne Greimas (1970).

14. Nous n'entrerons pas davantage ici dans l'étude formelle des tó-wèn.

\section{RÉSUMÉS}

Le proverbe dit «la parole pilée» correspond dans la culture des Gbaya de République Centrafricaine au niveau profond de la parole. Il s'agit d'un phénomène non de mémorisation mais de compréhension, qui constitue la principale voie d'accès au symbolisme. Cet article montre le cheminement qui permet l'acquisition de ces « paroles pilées » depuis l'enfance jusqu'à l'âge adulte.

The proverb which is known as "pounded words" corresponds, in Gbaya culture (Central African Republic), to the profound level of words. We are dealing with a phenomenon that involves not memorization but comprehension, which constitutes the principal access to symbolism. This article demonstrates the process by which one acquires these "pounded words" from childhood till adulthood.

\section{INDEX}

Mots-clés : Gbaya (langue), proverbe, langage -- acquisition

nomsmotscles Gbaya, Gbaya 'bodoé

Keywords : Central African Republic, Gbaya Languages, Gbaya, Proverb, Gbaya 'Bodoe Index géographique : République centrafricaine

Thèmes : anthropologie (Afrique) 